\title{
Many-body effects in a two-component, one-dimensional electron gas with repulsive, short-range interactions
}

\author{
B. Tanatar \\ Department of Physics, Bilkent University, Bilkent. 06533 Ankara, Turkey
}

Received 26 August 1997; revised manuscript received 5 November 1997; accepted for publication 9 January 1998

Communicated by J. Flouquet

\begin{abstract}
We investigate the correlation effects in a two-component, one-dimensional electron gas interacting via repulsive $\delta$ function interactions. Electron correlations are treated within the approximation scheme of Singwi, Tosi, Land, and Sjölander (STLS) which yields analytical expressions for the local-field corrections. The static structure factors, pair distribution functions, collective excitation modes, and interaction energy in a two-component system are discussed. (C) 1998 Elsevier Science B.V.
\end{abstract}

PACS: $71.10 .+\mathrm{x} ; 71.45 . \mathrm{Gm} ; 71.27 .+\mathrm{a}$

\section{Introduction}

One-dimensional (1D) electronic systems have attracted a lot of interest [1-3], because of their applicability to naturally occurring organic conductors, artificially fabricated semiconductor structures, and certain materials exhibiting superconductivity. The restrictions in the phase space of the 1D systems, stemming from the confinement in other spatial directions, result in a number of interesting effects, and present a rich structure. The study of many-body exchange and correlation effects in homogeneous quantum electron liquids is a field of active research and is of continuing interest because of current applications. The electrons interacting via the $1 / r$ Coulomb potential provide a suitable model for metals and doped semiconductors, which can also be a testing ground for various many-body theories. Recent technological advances have made it possible to effectively synthesize and manufacture $1 D$ systems with many striking experi- mental results. The random-phase approximation [4] (RPA) has been very successful in describing the dielectric properties of the interacting electron system with long-range Coulomb interaction in the high density limit. As the density of the electrons is lowered, the exchange and correlation effects become increasingly important, leading to many interesting physical phenomena. An approximation scheme to study the correlations in interacting electron systems has been introduced by Singwi et al. (STLS) [5] in terms of the local-field factors. The local fields take the repulsion hole around an electron into account to describe the correlation effects. The method has been extensively applied with great success to a number of physical problems $[5,6]$.

In a recent Letter, Gold [7] studied the exchangecorrelation effects in a $1 \mathrm{D}$ electron gas with shortrange interaction using the concept of local-field corrections. The short-range interaction is often considered for its analytical facility in a range of models 
to simulate more realistic problems. The correlation effects were described within the STLS approach [5] which yields analytic results for the various physical quantities of interest. The approximation scheme of STLS as applied to a 1D electron system gives good agreement with exact or field-theoretical results, provided the interaction strength parameter is not too large. The intense activity in the study of Luttinger liquids and the one-dimensional Hubbard model have advanced our understanding of the correlation effects in low-dimensional systems [8]. Gold [7] attempted to bridge the gap between continuum approaches (using homogeneous electron liquid and local-field corrections ideas) and the recent theoretical methods such as bosonization techniques $[9,10]$ and conformal-field theory [11]. One-dimensional fermions interacting via a repulsive $\delta$ function potential were treated by Yang [12] using the well-known Bethe ansatz method (usually applied to attractive interactions). Friesen and Bergersen [13] numerically solved Yang's equations [12] to calculate the groundstate energy of the system, and compared them with the STLS results. The ground-state energy and density response of a 1D fermion system with repulsive $\delta$ function interaction was also studied by Nagano and Singwi [14] within the ladder approximation.

Our aim in this Letter is to extend the calculations of Gold [7] to two-component, one-dimensional systems, the chief inspiration being two-band models as recently discussed in the literature [15]. We use repulsive, short-range interactions with different strengths to describe the interactions between the same and different species in a two-component system. The correlation effects are similarly described by the multicomponent generalization [16] of the STLS approach. To keep the discussion at the same level of transparency as that of Gold [7], we introduce a generalization of the analytic static structure factors for the two-component problem. Our second goal is to see to what extent a two-component system is described by analytic structure factors, which have a close analogy to bosonic systems. These techniques facilitate the analysis of physical quantities, such as collective modes. Multi-component systems often display new and interesting phenomena usually absent in the single-component counterparts, as in doublelayer electron systems [17]. The correlation effects in double-wire electron-electron and electron-hole sys- tems with realistic Coulomb interactions (long-range) have recently been reported [18]. The short-range interactions studied in this work should complement the previous studies, and also make contact with the cal culations involving field-theoretical techniques.

\section{Theory and model}

The two-component, one-dimensional electron system we study is characterized by the interaction potentials $V_{11}$ and $V_{12}$. We assume repulsive $\delta$ function (contact) potentials in the $r$ space, which yield constant values $V_{11}$ and $V_{12}\left(V_{11}, V_{12}>0\right)$ in the $q$ space. We can regard $V_{11}$ to represent the interaction between like particles, and $V_{12}$ to represent interaction between different species. Their numerical values may be related to the appropriate scattering lengths. If a two-band model is considered, $V_{11}$ and $V_{12}$ describe the intra- and inter-band interactions, respec tively. Assuming the same mass and linear density for both species, we define the dimensionless parameters $\gamma_{1}=m V_{11} / n$ and $\gamma_{2}=m V_{12} / n$ to describe the strength of the interactions. Note that the equal-mass assumption is also used in various integrable two-band models [15]. The electron density is related to the Fermi wave number by $n=2 k_{\mathrm{F}} / \pi$. Furthermore, adopting the Fermi-liquid picture we employ the parabolic dispersion relation $\epsilon_{4}=q^{2} / 2 m$ for the single-particle energies.

In the many-body description of electron liquids, the wave vector- and frequency-dependent density response function $\chi(q, \omega)$ plays a central role, characterizing the response of the system to external longitudinal perturbations. In the case of multi-component systems, which are formally similar to multi band sys. tems, the response is expressed as a matrix equation [16]. Within the STLS approximation the response to an external field is formulated as that of a noninteracting system responding to an effective potential, which in the equal-density, equal-mass, twocomponent system becomes $\varphi_{i j}(q)=V_{i j}\left[1-G_{i j}(q)\right]$, where $i$ and $j$ label the species. The local-field factors $G_{i j}(q)$ describe the short-range correlation effects neglected by the RPA. The integral expressions for $G_{i j}(q)$ involve the static structure factors $S_{i j}(q)$, which follow from the assumption that the two-particle distribution function may be decoupled as a product 
of two one-particle distribution functions multiplied by the pair-correlation function [5],

$G_{i j}=\frac{1}{2} \int_{0}^{\infty} \mathrm{d} x\left[\delta_{i j}-S_{i j}(x)\right]$.

Evidently, $G_{i j}$ have no $q$ dependence within the STLS scheme when applied to contact potentials. It has been shown [14] that STLS approximate the full local-field factor by its high frequency $(\omega \rightarrow \infty)$ limit.

We next introduce the generalization of analytic expressions for the static structure factors in a twocomponent system. The boson-like static structure factors may be obtained within the mean-spherical approximation $[19,20]$ (MSA), which replaces the noninteracting response function by the expression $\chi_{0}^{\mathrm{MSA}}(q, \omega)=2 n \epsilon_{q} /\left\{\omega^{2}-\left[\epsilon_{q} / S_{0}(q)\right]^{2}\right\}$, where $\epsilon_{q}=$ $q^{2} / 2 m$, and $S_{0}(q)$ is the static structure factor of the noninteracting electron gas in $1 \mathrm{D}$, i.e. $S_{0}(q)=q / 2 k_{\mathrm{F}}$, for $q<2 k_{\mathrm{F}}$, and $S_{0}(q)=1$, for $q>2 k_{\mathrm{F}}$. In $\chi_{0}^{\mathrm{MSA}}$ the particle-hole pair continuum is approximated by a collective mode with energy $\epsilon_{q} / S_{0}(q)$, similar to the Feynman excitation spectrum for bosons [19]. Solving for the collective modes from the matrix expression [16] of $\chi(q, \omega)$, and identifying them with the excitation spectrum of the Feynman type, we obtain

$$
\begin{aligned}
& S_{11,12}(q)=\frac{1}{2}\left[x \left(\frac{x^{2}}{\left[S_{0}(x)\right]^{2}}+\frac{16 \gamma_{1}\left(1-G_{11}\right)}{\pi^{2}}\right.\right. \\
& \left.\quad-\frac{16 \gamma_{2}\left(1-G_{12}\right)}{\pi^{2}}\right)^{-1 / 2} \\
& \quad \pm\left[x \left(\frac{x^{2}}{\left[S_{0}(x)\right]^{2}}+\frac{16 \gamma_{1}\left(1-G_{11}\right)}{\pi^{2}}\right.\right. \\
& \left.\left.+\frac{16 \gamma_{2}\left(1-G_{12}\right)}{\pi^{2}}\right)^{-1 / 2}\right],
\end{aligned}
$$

where $x=q / k_{\mathrm{F}}$. The above expressions may also be obtained by the frequency integrals of the fluctuationdissipation theorem within the MSA. These equations provide closed-form expressions for the static structure factors in terms of the local-field corrections. They are the generalization of the expression given by Gold [7] for the single-component case, and may be regarded as generalized MSA expressions using the terminology of Iwamoto et al. [19]. Such boson-like static structure factors have proved very useful in many applications of multi-component Fermi liquids [21].

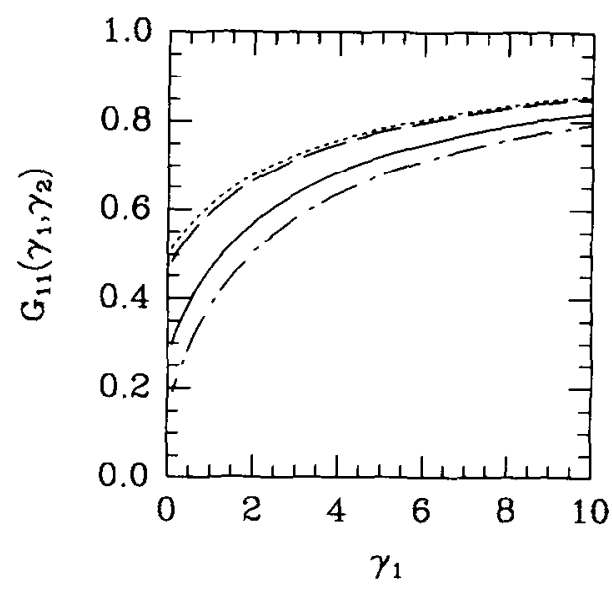

Fig. 1. The local-field correction $G_{11}$ as a function of the coupling strength $\gamma_{1}$, for $\gamma_{2}=0$ (dotted line), $\gamma_{2}=1$ (dashed line), $\gamma_{2}=5$ (solid line), and $\gamma_{2}=10$ (dot-dashed line).

Solving the set of coupled equations for the static structure factors and local-field corrections, we find

$$
\begin{gathered}
G_{11,12}= \pm \frac{1}{2}\left\{\left(1+\frac{A+B}{4}\right)^{1 / 2} \pm\left(1+\frac{A-B}{4}\right)^{1 / 2}\right. \\
-\frac{1}{2}\left[\left(1+\frac{A-B}{4}\right)^{1 / 2} \pm\left(1+\frac{A+B}{4}\right)^{1 / 2}\right] \\
\left.\times\left(1+\frac{A-B}{4}\right)^{-1 / 2}\left(1+\frac{A+B}{4}\right)^{-1 / 2}\right\},
\end{gathered}
$$

where $A=16 \gamma_{1}\left(1-G_{11}\right) / \pi^{2}$ and $B=16 \gamma_{2}(1-$ $\left.G_{12}\right) / \pi^{2}$. In the next section, we discuss our results, using the solutions to the above equations for $G_{i j}$.

\section{Results and discussion}

For a given set of interaction strengths $\gamma_{1}$ and $\gamma_{2}$, we can solve the coupled equations (Eq. (3)) to obtain the local-field corrections $G_{11}\left(\gamma_{1}, \gamma_{2}\right)$ and $G_{12}\left(\gamma_{1}, \gamma_{2}\right)$. As $\gamma_{2} \rightarrow 0$, Eq. (3) gives $G_{12}=0$, and we recover the single-component results of Gold [7]. In Figs. 1 and 2, we show the numerical calculations of the local-field corrections. The dependence of $G_{11}$ on $\gamma_{1}$ is affected more as the interaction strength $\gamma_{2}$ is increased. $G_{12}$, on the other hand, remains roughly constant as a function of $\gamma_{1}$, for different values of $\gamma_{2}$. The non-zero values of $G_{12}$ indicate that as the interaction with the second species is turned on, the correlations among the like and unlike particles are 


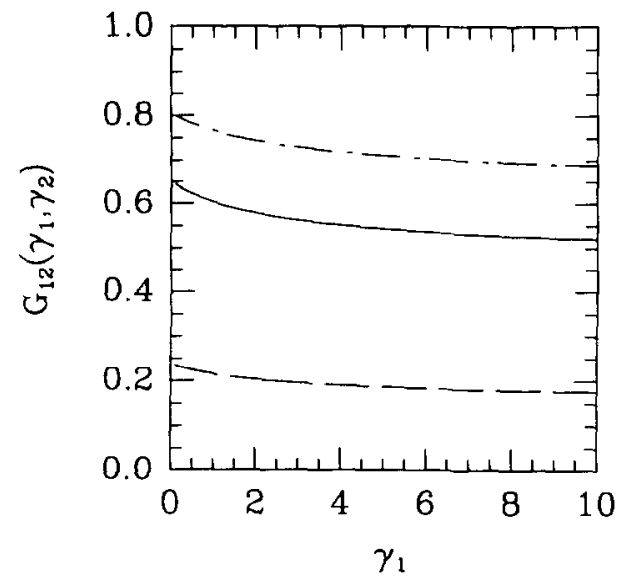

Fig. 2. The local-field correction $G_{12}$ as a function of the coupling strength $\gamma_{1}$, for $\gamma_{2}=1$ (dashed line), $\gamma_{2}=5$ (sulid line), and $\gamma_{2}=10$ (dot-dashed line)

further increased compared to the single-component case.

Using the local-field corrections obtained above, we calculate the static structure factors $S_{11}(q)$ and $S_{12}(q)$ as shown in Fig. 3. For $\gamma_{1}=1$ and $\gamma_{2}=0$, we have the structure factor of a one-component system (dotted line), with the characteristic behavior of a onedimensional Fermi system at small coupling. As the interaction with the second component is switched on $\left(\gamma_{2}=1\right), S_{11}(q)$ slightly increases and $S_{12}(q)$ appears with a peak at $2 k_{\mathrm{F}}$ (dashed lines). With increasing $\gamma_{2}$, the magnitudes of $S_{11}(q)$ and $S_{12}(q)$ increase (solid lines). In particular, $S_{11}(q)$ exhibits a sharp peak at $2 k_{\mathrm{F}}$, not present in single-component systems.

Once the static structure factors are obtained, we can turn our attention to the pair distribution functions. The pair distribution functions $g_{i j}(r)$ give the probability of finding an electron (of species $i$ ) at a distance $r$, when there is another electron (of species $j)$ at the origin. From the knowledge of $S_{i j}(q)$, we obtain $g_{i j}(r)$ by the Fourier transform

$g_{i j}(r)=1+\frac{1}{n} \int \frac{\mathrm{d} q}{2 \pi} \cos (q r)\left[S_{i j}(q)-\delta_{i j}\right]$.

In particular, the pair distribution functions at the origin are given by $g_{i j}(r=0)=1-G_{i j}\left(\gamma_{1}, \gamma_{2}\right)$. From the behavior of the local-field factors discussed in Figs. 1 and 2 , we observe that $g_{i j}(0)$ remain positive for all coupling strengths. This is in contrast to the situation for systems interacting via a long-range Coulomb

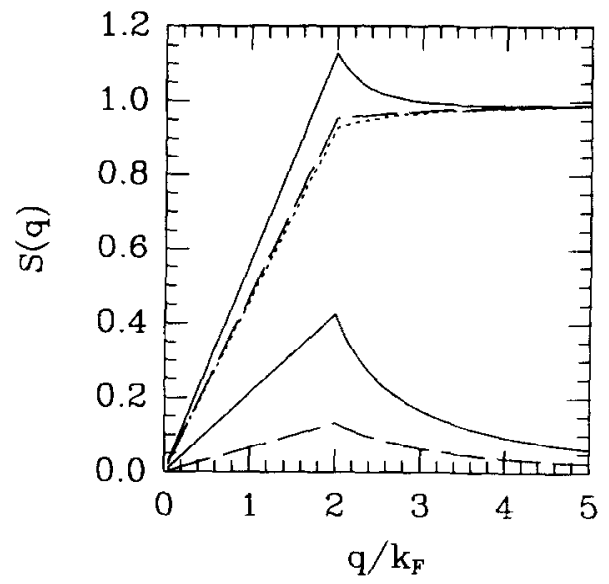

Fig. 3. The static structure factors $S_{11}(q)$ (top curves) and $S_{12}(q)$ (bottom curves) at $\gamma_{1}=1$. The dutted line indicates the single-component result, whereas dashed and solid lines are for $\gamma_{2}=1$ and 5 , respectively.

interaction. Typically, the STLS approximation gives rise to the unphysical result of $g(0)<0$ for moderate coupling strengths $[5,6]$.

The collective modes of the two-component system under study is obtained from the solutions of $[1$ $\left.V_{11}\left(1-G_{11}\right) \chi_{0}(q, \omega)\right]^{2}-\left[V_{12}\left(1-G_{12}\right) \chi_{0}(q, \omega)\right]^{2}=$ 0 . If we use the non-interacting response function for one-dimensional fermions, the ensuing plasmon modes (scaled with Fermi energy $E_{\mathrm{F}}$ ) are given by

$$
\begin{aligned}
& \frac{\omega_{1,2}(q)}{E_{\mathrm{F}}}=2 x\left[1+\frac{x^{2}}{4}\right. \\
& \left.+x \operatorname{coth}\left(\frac{\pi^{2} x}{4\left[\gamma_{1}\left(1-G_{11}\right) \pm \gamma_{2}\left(1-G_{12}\right)\right]}\right)\right]^{1 / 2},
\end{aligned}
$$

which is the generalization of single-component dispersion. However, it might be argued that a more consistent approach is to use the mean-spherical approximation expression $\chi_{0}^{\mathrm{MSA}}(q, \omega)$, which yields

$$
\begin{aligned}
& \omega_{1,2}(q) / E_{\mathrm{F}}=\left[\left(\frac{x^{2}}{S_{0}(x)}\right)^{2}+\frac{16}{\pi^{2}}\left[\gamma_{1}\left(1-G_{11}\right)\right.\right. \\
& \left.\left. \pm \gamma_{2}\left(1-G_{12}\right)\right] x^{2}\right]^{1 / 2} .
\end{aligned}
$$

It turns out that both approaches give the same result, 


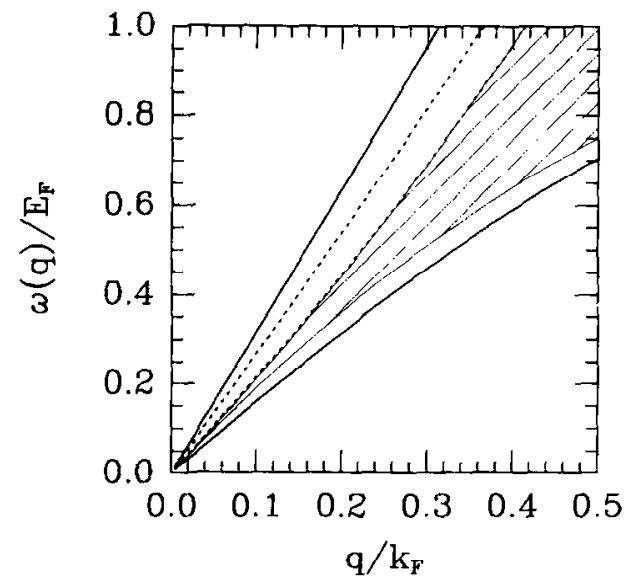

Fig. 4. The collective modes of a two-component system at $\gamma_{1}=5$. The dotted and solid lines are for $\gamma_{2}=1$, and $\gamma_{2}=5$, respectively. The hatched region indicates the particle-hole continuum of a 1D electron gas.

$$
\begin{aligned}
& \frac{\omega_{1.2}(q \rightarrow 0)}{\omega_{ \pm}(q \rightarrow 0)} \\
& \quad \approx\left(1+\frac{4}{\pi^{2}}\left(\gamma_{1}\left(1-G_{11}\right) \pm \gamma_{2}\left(1-G_{12}\right)\right)\right)^{1 / 2}
\end{aligned}
$$

in the long-wavelength limit, and deviations set in for $q \gtrsim 0.5 k_{\mathrm{F}} \cdot \omega_{ \pm} / E_{\mathrm{F}}=\left|x^{2} \pm 2 x\right|$ determine the boundaries of the particle-hole excitation rcgion in one-dimensional systems. $\omega_{1}(q)$ and $\omega_{2}(q)$ are the dispersion relations for the collective charge excitations of the system responding to the in-phase and outof-phase charge oscillations. They correspond to the charge and inter-band excitations as discussed within the Luttinger liquid description [3]. Li et al. [22]. have shown that the Fermi liquid theory in ID gives the same dispersion relation as that of the Luttinger liquid theory, in the long-wavelength limit. We surmise that similar results should hold in the multi-component case as well. Fig. 4 displays the collective excitation modes in a two-component system (based on Eq. (5)) for $\gamma_{1}=5$. When the interaction strength $\gamma_{2}$ is small $\left(\gamma_{2}=1\right.$ in Fig. 4$)$, we observe that both plasma modes are above the single-particle excitation region, $\omega_{2}(q)$ getting very close to $\omega_{+}$. With increasing $\gamma_{2}$, the separation between the modes become more noticeable. In the example shown in Fig. 4 (for $\gamma_{2}=5$ ), $\omega_{2}(q)$ falls below the lower boundary of the single-particle excitation region.

The exact ground-state energy of a one-dimensional

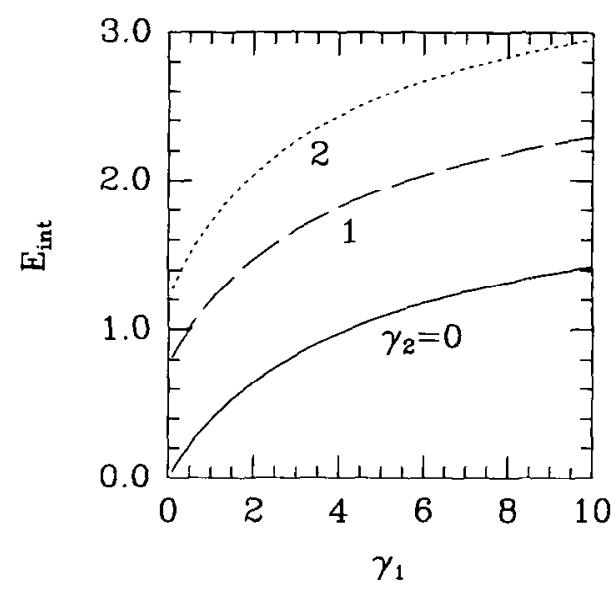

Fig. 5. The interaction energy in a two-component system as a function of the coupling strength $\gamma_{1}$, for $\gamma_{2}=0$ ( single-component system, solid line), $\gamma_{2}=1$ (dashed line), $\gamma_{2}=2$ (dotted line)

electron gas with repulsive short-range interactions was numerically calculated by Friesen and Bergersen [13] based on the integral equations of Yang [12]. Nagano and Singwi [14] showed that the ladder sums approximate the exact energy quite well even for large coupling constant strengths. It is known that the STLS results for the energy are only good for moderate $\gamma_{1}$ values (i.e., $\gamma_{1} \lesssim 10$ ), still an improvement over the RPA. We expect similar conclusions to hold in a two-component systeml. The interaction energy in a two-component system is given by

$$
\begin{aligned}
& E_{\text {int }}\left(\gamma_{1}, \gamma_{2}\right)=\left(\frac{n^{2}}{2 m}\right) 2\left[\gamma_{1}\left(1-G_{11}\right)\right. \\
& \left.\quad+\gamma_{2}\left(1-G_{12}\right)\right],
\end{aligned}
$$

where we have also included the Hartree contributions to the energy. In Fig. 5 we show the interaction energy as a function of $\gamma_{1}$, for various values of $\gamma_{2}$. Within the STLS approximation scheme, $E_{\text {int }}$ is a monotonic function of $\gamma_{1}$ and $\gamma_{2}$, and does not exhibit a peak structure as in the exact result or in the ladder approximation [14]. Schlottmann [23] have studied an integrable model consisting of two bands of electrons with local repulsive interactions. Because the same interaction strength is used for intra- and inter-band couplings in this model, it is somewhat difficult to make a direct comparison.

In this Letter we have studied the short-range correlation effects in a two-component, one-dimensional electron system, interacting via short-range (contact) 
interactions. We have used the STLS approximation scheme to treat the static correlations between the electrons. The two-component system, with local repulsive interactions can be useful to understand some properties of high- $T_{\mathrm{c}}$ cuprates, since the $3 d_{x^{2}-y^{2}}$ and $3 d_{z^{2}}$ orbitals (considered within a two-band model) are thought to play an important role. We mention in passing that our approach may further be generalized to include attractive interactions, and in particular a simple model [3] for excitons may be developed by considering an electron-hole system. We have introduced analytical expressions for the static structure factors in a two-component system based on the boson analogy $[7,19,21]$. The present analytical approximations to the local-field factors in two-component (or two-band) electron systems provide a simplc alternative to the computationally more demanding full solution of the STLS equations. The Luttinger liquid and Hubbard model have exact solutions [2] in 1D. Making direct comparisons of our calculations with these results, however, we have to keep in mind the discrete and continuum model descriptions, and linearized versus parabolic single-particle dispersions employed in the respective models. The local-field factor introduced to describe the corrections to the RPA is finding its way to the modern description of interacting Fermi systems [24].

The 1D electron systems are considered to be nonFermi liquids $[8,9]$. Quasi-one-dimensional electron systems as occur in semiconductor structures, on the other hand, are successfully described by an interacting system of electrons within the Fermi liquid theory [25]. Experimental manifestations of non-Fermi liquid behavior in organic $1 \mathrm{D}$ materials are a subject of current interest [26]. We believe that our calculations based on the Fermi liquid picture may still be applicable to certain physical situations.

Correlation effects in a one-dimensional Bose gas, interacting with a repulsive $\delta$ function potential were also discussed by Gold [27]. Comparison with the exact results of Lieb and Liniger [28] showed better agreement than the Bogoliubov approximation predictions which neglects correlation effects. Our generalized formulation can be applied to a two-component, one-dimensional Bose gas, with appropriate modifications.

In summary, we have presented a two-component generalization of many-body calculations in a one- dimensional electron gas with repulsive, short-range interactions. With the analytical results obtained for the local-field corrections, the pair distribution function, collective modes, and interaction energy of the system are discussed.

\section{Acknowledgement}

This work is partially supported by the Scientific and Technical Research Council of Turkey (TUBITAK) under Grant No. TBAG-AY/123. We thank Dr. M.Z. Gedik, Dr. O. Tekman, and Dr. N. Bulut for fruitful discussions.

\section{References}

[1] J. González, M.A. Martín Delgado, G. Sicrra, A.H Vozmediano, eds., Quantum Electron Liquids and High- $T_{L}$ Superconductivity (Springer, Berlin, 1995).

[2] D.C. Mattis, The Many-Body Problem (World Scientific. Singapore, 1993).

[3] P. Schlottmann, Int. J. Mod. Phys. B 11 (1997) 355

[4] D. Pines, P. Nozières, The Theory of Quantum Liquids (Benjamin, New York, 1966).

[5] K.S. Singwi, M.P. Tosi, R.H. Land, A. Sjölander, Phys. Rev 179 (1968) 589;

K.S. Singwi, M.P. Tosi, Solid State Physics 36 (1981) 177

[6] P. Jena. R. Kalia. P. Vashishta. M.P. Tosi. eds.. Correlations in Electronic and Atomic Fluids (World Scientific, Singapore. 1990).

[7] A. Gold. Phys. Rev. B 55 (1997) 9470

[8] J. Sólyom, Adv. Phys. 28 (1979) 209.

[9] F.D.M. Haldane. Phys. Rev. Lett. 47 (1981) 840; J. Phys. C 14 (1981) 2585

[10] J. Voit, Phys. Rev. B 47 (1993) 6740.

[1]] H. Frahm, V.E. Korepin, Phys. Rev. B 42 (1990) 10553 and references therein

[12] C.N. Yang. Phys. Rev. Lett. 19 (1967) 1312

[13] W.I. Friesen, B. Bergersen, J. Phys. C 13 (1980) 6627

[14] S. Nagano, K.S. Singwi, Phys. Rev. B 27 (1983) 67.32; 28 (1983) 6286

[15] P. Schlottmann, Phys. Rev. Lett. 68 (1992) 1916; 69 (1992) 2396; J. Phys. Condens. Matter 7 (1995) 111.

[16] L. Zheng, A.H. MacDonald, Phys. Rev. B 49 (1994) 5522 L. Iiu, L. Świerkowski, D. Neilson, J. Szymański, Phys. Rev. B 53 (1996) 7923;

J. Szymánski, L. Świerkowski. D. Neilson, Phys. Rev. B 50 (1994) 11002.

[17] S. Das Sarma, A. Pinczuk, eds.. Perspectives in Quantum Hall Effects: Novel Quantum Liquids in Low-Dimensional Seniconductor Structures (Wiley, New York, 1996).

[18] N. Mutluay, B. Tanatar. Phys. Rev. B 55 (1997) 6697; J.S. Thakur, D. Neilson, Phys. Rev. B 56 (1997). 
[19] N. Iwamoto, D. Pines, Phys. Rev. B 29 (1991) 3924;

N. Iwamoto, E. Krotscheck, D. Pines, 29 (1984) 3936;

R.F. Bishop, K.H. Lührmann, 26 (1982) 5523.

[20] A. Gold, L. Calmels, Phys. Rev. B 48 (1993) 11622

[21] C.E. Campbell, J.G. Zabolitzky, Phys. Rev. B 29 (1984) I23.

122] Q.P. Li, S. Das Sarma, R. Joynt, Phys. Rev. B 45 (1992) 13713.

[23] P. Schlottmann, Phys. Rev. B 49 (1994) 6132.

[24] P. Kopietz, J. Hermisson, K. Schönhammer, Phys. Rev.
B 52 (1995) 10877;

M.R. Hedayati, G. Vignale, 40 (1989) 9044.

[25] B.Y.-K. Hu, S. Das Sarma, Phys. Rev. Lett. 68 (1992) 1750; Phys. Rev. B 48 (1993) 5469.

[26] G.-H. Gweon, J.W. Allen, R. Claessen, J.A. Clack, D.M. Poirier, P.J. Benning, C.G. Olson, W.P. Ellis, X.-Y. Zhang, L.F. Schneemeyer, J. Marcus, C. Schlenker, J. Phys. Condens. Matter 8 (1996) 9923.

[27] A. Gold, Z. Phys. B 91 (1993) 387.

[28] E.H. Lieb, W. Liniger, Phys. Rev. 130 (1963) 1605. 1616. 